\title{
ADDITIONS TO THE MOSS FLORA OF PENINSULAR INDIA FROM THE WESTERN GHATS
}

\author{
R. Sreebha, K. C. Kariyappa and A. E. D. Daniels* \\ Bryology Laboratory, Department of Botany and Research Centre, Scott Christian College, \\ Nagercoil-629 003, Tamil Nadu, India; *E-mail: dulipdaniels@yahoo.co.uk
}

(Received 1 June, 2016; Accepted 30 June, 2016)

Two Asian mosses Entodontopsis setschwanica (Broth.) W. R. Buck et R. R. Ireland and Mitthyridium cardotii (M. Fleisch.) H. Rob., so far known from the Eastern Himalaya and Northeast India, respectively for India, are added here to the moss flora of Peninsular India from the Western Ghats. Brief descriptions with illustrations are provided.

Key words: Anamalais, Entodontopsis setschwanica, Mitthyridium cardotii, Western Ghats

\section{INTRODUCTION}

Since knowledge on cryptogams is still far from adequate, studies on the bryoflora of the Indira Gandhi National Park in Anamalais was initiated a few years ago and important findings have been published as and when found. In the present contribution two Asian mosses Entodontopsis setschwanica (Broth.) W. R. Buck et R. R. Ireland and Mitthyridium cardotii (M. Fleisch.) H. Rob., so far known from the Eastern Himalaya and Northeast India, respectively for India, are added here to the moss flora of Peninsular India from the Western Ghats. The species dealt with are provided with the correct name, basionym/synonyms, if any, brief descriptions with illustrations, notes on habitat and distribution.

Entodontopsis setschwanica (Broth.) W. R. Buck et R. R. Ireland Beih. Nova Hedwigia 41: 105 (1985)

(Figs 1-12)

Basionym: Stereophyllum setschwanicum Broth., Akad. Wiss. Wien Sitzungsber., Math.Naturwiss. Kl., Abt. 1, 133: 581 (1924); Chopra, R. S., Taxon. Indian Moss, p. 487 (1975); Gangulee, Moss. E. India 3(8): 1817, f. 921 (1980); J. Lal, Checklist Indian Moss., p. 127 (2005). - Type: China, Setschwan Province, 1350 to 1450 m, 28 September, 1914, Handel-Mazzetti, H. 5330 (P). 


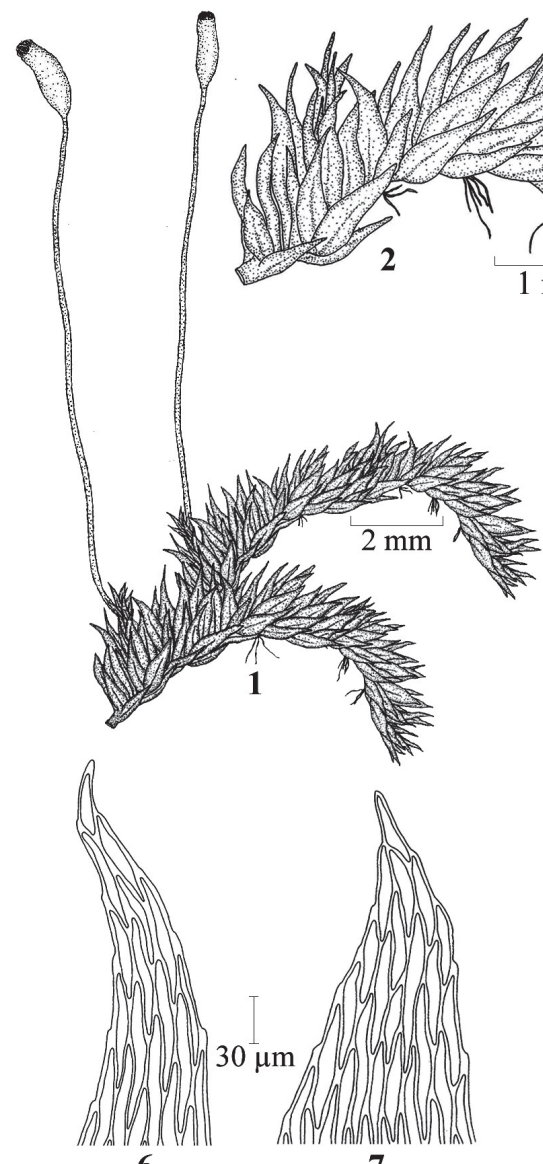

6 7
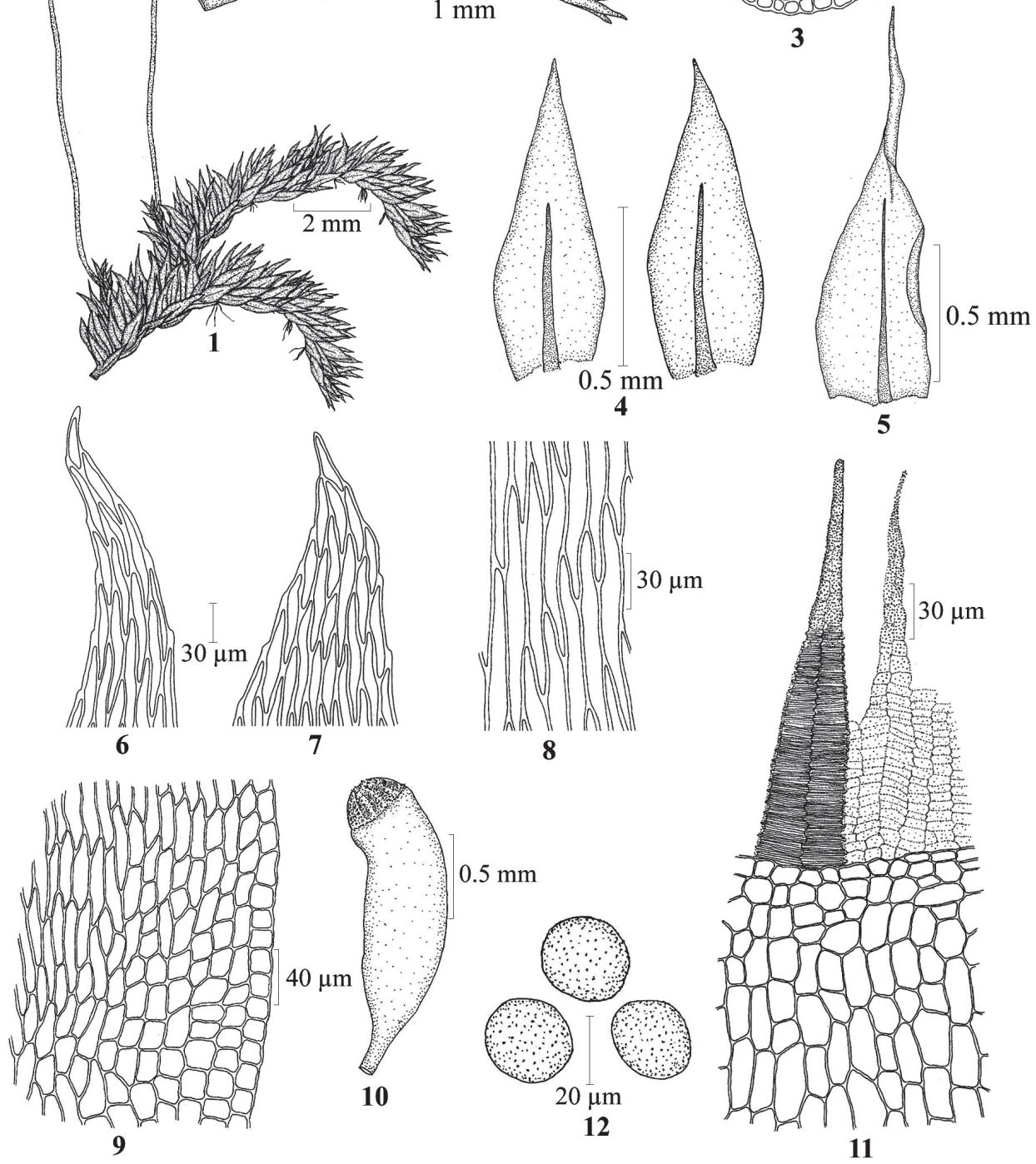

11

Figs 1-12. Entodontopsis setschwanica (Broth.) W. R. Buck et R. R. Ireland $-1=$ sporophytic plant, $2=$ portion of plant, $3=$ cross-section of stem, $4=$ leaves, $5=$ perichaetial leaf, $6-7=$ leaf apical cells, $8=$ leaf median cells, $9=$ leaf basal cells, $10=$ capsule, $11=$ peristome teeth, 12 = spores (drawn from Sreebha, R. and Kariyappa, K. C. 9640 p. p.) 
Plants robust, glossy, yellow-green. Stems creeping, rooted by rhizoidal clusters, $5-15 \mathrm{~mm}$ long, sparsely irregularly branched, $0.18-0.2 \times 0.2-0.22 \mathrm{~mm}$ in cross-section, ovate, without a central strand; cortex 2- or 3-layered; cells 8-16 × 6-12 mm, thick-walled, rounded-quadrate; medullary ones 10-36 $\times$ 8-20 mm, quadrate-hexagonal, thin-walled. Leaves 2.5-3 × 1-1.5 mm, complanate, erect, pressed to one side, symmetric, ovate-lanceolate, entire at margin, sometimes recurved on sides, acute to acuminate at apex; apical and median cells linear elongate; apical ones 32-88 × 6-10 $\mu$ m; median ones 120-140 × 8-10 $\mu \mathrm{m}$, basal ones 12-40 × 8-14 $\mu \mathrm{m}$, quadrate to rectangular, smooth; costa single, more than $1 / 2$ as long as leaf. Sporophytes lateral. Perichaetial leaves erect, 1.3-1.8 $\times 0.32-0.48 \mathrm{~mm}$, broad, acuminate. Setae erect, $8-10 \mathrm{~mm}$ high, smooth. Capsules horizontal to inclined, $1.5-1.8 \times 0.5-0.6 \mathrm{~mm}$, ovoid-cylindrical. Peristome teeth double; exostome $0.1-0.12 \times 0.032-0.04 \mathrm{~mm}$, with fine horizontal striations below, papillose at apex; endostome $0.1-0.11 \times 0.03-0.042$ $\mathrm{mm}$, hyaline. Spores 18-20 mm, globose, smooth, brown.

Habitat: Lignicolous, in degraded moist deciduous and evergreen forests, $800-1250 \mathrm{~m}$.

Distribution: China, Nepal and India: Eastern Himalaya (W Bengal) and Western Ghats of Tamil Nadu (Coimbatore).

Specimens examined: India, Western Ghats, Tamil Nadu, Coimbatore Dist., Anamalais, Topslip, Alt.: ca 820 m a.s.l., Daniels, A. E. D. and Kariyappa, K. C. (9641 p. p., 9679 p. p., 9680 p. p., 9681 p. p.), 23.9.2014 (SCCN); Valparai, Alt.: ca 1250 m a.s.l., Sreebha, R. and Kariyappa, K. C. (9849 p. p.), 4.2.2015 (SCCN).

Mitthyridium cardotii (M. Fleisch.) H. Rob.

Phytologia 32: 432 (1975)

(Figs 13-19)

J. Lal, Checklist Indian Moss., p. 91 (2005).

Basionym: Thyridium cardotii M. Fleisch., Musc. Buitenzorg. 1: 228. f. 34 (1904); Bruehl, Rec. Bot. Surv. India 13(1): 32 (1931); Chopra, R. S., Taxon. Indian Moss, p. 104 (1975); Gangulee, Moss. E. India 1(3): 583, f. 297 (1972). 三 Calymperes cardoti (M. Fleisch.) Paris, Index Bryol. (ed. 2), 1: 276 (1904). इ Syrrhopodon cardotii (M. Fleisch.) Broth., Nat. Pflanzenfam I(3): 1188 (1909). इ Mitthyridium fasciculatum Hook. et Grev. subsp. cardotii (M. Fleisch.) B. C. Tan et L. T. Ellis, Bull. Nat. Hist. Mus. London, Bot. 29: 25 (1999). - Type: Indonesia (West-Java), Buitenzorg, Botanic Garden, Massart; Krawang, Goenoeng Parang, 800 m (F).

Plants densely caespitose, 3-4 cm tall, yellowish-green. Stems ca $0.22 \times$ $0.14 \mathrm{~mm}$ in cross-section, circular to ovate, without a central strand; cortex 1- or 2-layered; cells 8-12 × 10-14 mm, thin-walled; medullary ones 12-24 × 10-16 mm, thin-walled. Leaves curled when dry, erect to patent when wet, $1.7-2.8 \times 0.4-0.7 \mathrm{~mm}$, obovate to lingulate, entire above, faintly toothed below at margin, acute; cells rounded to quadrate, multipapillate; apical and median 
ones 8-10 × 7-8 $\mu \mathrm{m}$; basal ones at margin 16-40 × 4-8 $\mu \mathrm{m}$; those of cancellina 13-18-rowed on either side of costa, 28-36 × 12-16 $\mu \mathrm{m}$, pellucid, elongate, rectangular, smooth, bordered by cartilaginous cells; cartilaginous border cells 10-rowed, narrowing down to 3 rows; costa percurrent. Sporophyte not seen.

Habitat: Corticolous on Camellia sinensis (L.) O. Kuntze (Theaceae) in tea plantations, ca $920 \mathrm{~m}$.

Distribution: Cambodia, Indonesia, Myanmar, Vietnam and India: Northeast India (Meghalaya) and Western Ghats of Tamil Nadu (Coimbatore).

Specimen examined: India, Western Ghats, Tamil Nadu, Coimbatore Dist., Anamalais, Valparai, Anali estate, Alt.: ca 920 m a.s.l., Daniels, A. E. D. (8927), 13.8.2012 (SCCN).

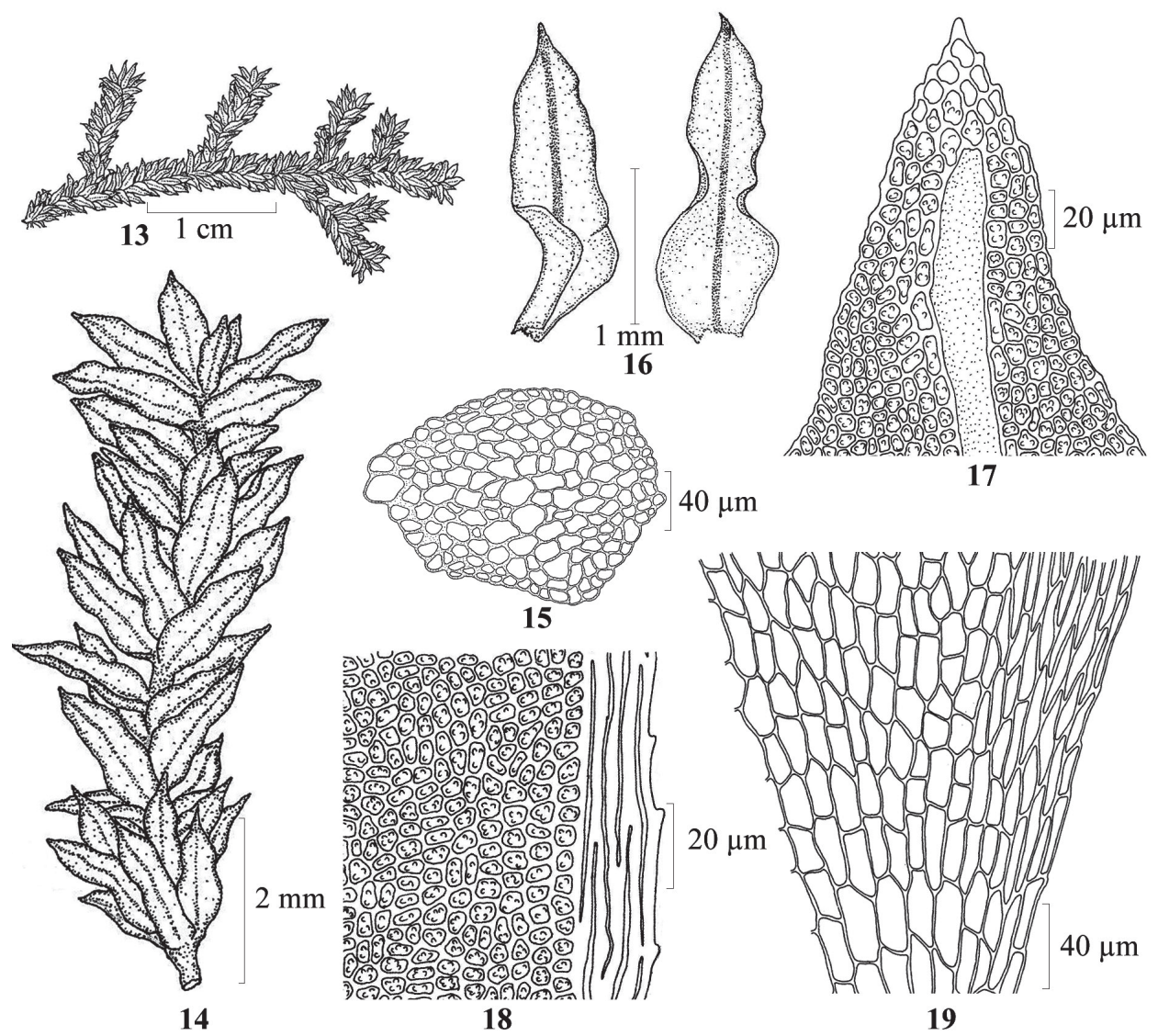

Figs 13-19. Mitthyridium cardotii (M. Fleisch.) H. Rob. $-13=$ plant, $14=$ portion of plant, $15=$ cross-section of stem, $16=$ leaves, $17=$ leaf apical cells, $18=$ leaf median cells with marginal cells, $19=$ leaf basal cells (drawn from Sreebha, R. and Kariyappa, K. C. 9640 p. p.) 
Acknowledgements - We thank the Tamil Nadu State Forest Department for permission to explore the study area. AEDD thanks the Department of Science and Technology (DST), New Delhi, for financial assistance and the Principal, Scott Christian College, for facilities.

\section{REFERENCES}

Bruehl, P. (1931): A census of Indian mosses with analytical keys to the genera. - Rec. Bot. Surv. India 13(1): 1-135.

Buck, W. R. and Ireland, R. R. (1985): A reclassification of the Plagiotheciaceae. - Nova Hedwigia 41: 89-125.

Chopra, R. S. (1975): Taxonomy of Indian mosses. - CSIR, New Delhi, India, 631 pp.

Gangulee, H. C. (1969-1980): Mosses of Eastern India and adjacent regions. 1-3 (Fasc. 1-8). Calcutta, India, $2142 \mathrm{pp}$.

Lal, J. (2005): A checklist of Indian mosses. - Bishen Singh Mahendra Pal Singh, Dehra Dun, India, $164 \mathrm{pp}$.

Robinson, H. (1975): A new name for the moss genus, Thyridium. - Phytologia 32: 432-435. 